\title{
A Methodology for Calculating Greenhouse Effect of Aircraft Cruise Using Genetic Algorithm-Optimized Wavelet Neural Network
}

\author{
Yong Tian $\mathbb{D}^{1},{ }^{1}$ Lina $M a \mathbb{D}^{1},{ }^{1}$ Songtao Yang $\mathbb{D},{ }^{2}$ and Qian Wang $\mathbb{D}^{1}$ \\ ${ }^{1}$ College of Civil Aviation, Nanjing University of Aeronautics and Astronautics, Nanjing 211100, China \\ ${ }^{2}$ School of Mechanical Engineering, University of Leeds, Leeds, LS2 9JT, UK \\ Correspondence should be addressed to Lina Ma; malinacca@nuaa.edu.cn
}

Received 21 July 2020; Revised 2 September 2020; Accepted 8 October 2020; Published 29 October 2020

Academic Editor: Min Xia

Copyright (c) 2020 Yong Tian et al. This is an open access article distributed under the Creative Commons Attribution License, which permits unrestricted use, distribution, and reproduction in any medium, provided the original work is properly cited.

Reliable assessment on the environmental impact of aircraft operation is vital for the performance evaluation and sustainable development of civil aviation. A new methodology for calculating the greenhouse effect of aircraft cruise is proposed in this paper. With respect to both cruise strategies and wind factors, a genetic algorithm-optimized wavelet neural network topology is designed to model the fuel flow-rate and developed using the real flight records data. Validation tests demonstrate that the proposed model with preferred network architecture can outperform others investigated in this paper in terms of accuracy and stability. Numerical examples are illustrated using 9 flights from Beijing Capital International Airport to Shanghai Hongqiao International Airport operated by Boeing 737-800 aircraft on October 2, 2019, and the generated fuel consumption, $\mathrm{CO}_{2}$ and $\mathrm{NO}_{\mathrm{x}}$ emissions as well as temperature change for different time horizons can be effectively given through the proposed methodology, which helps in the environmental performance evaluation and future trajectory planning for aircraft cruise.

\section{Introduction}

As a typical manifestation of the negative externalities of air transport, environmental impact is easily overlooked in management decisions, while it greatly affects the sustainability of operation. With the prosperity of global air transport industry, the dramatically increasing flights have resulted in prominent environmental issues [1]. Statistically, aviation is responsible for $13 \%$ of fossil fuel consumption related to transportation, $2 \%$ of the anthropogenic carbon dioxide emissions [2], and $24 \%$ of global nitrogen oxide emissions [3], and the climatic change caused by it will rise $3 \%-11 \%$ in the next 30 years [4]. Meanwhile, a further increase of these figures can be foreseen for the expected growth of global air traffic at an annual rate of $4.8 \%$ from 2011 to 2030 [5]. Under the determination in promoting green civil aviation development of the international community, it is imperative to focus on and study about the environmental impact caused by aircraft operation.
In order to address and evaluate the aviation environmental impact, current studies are conducted mostly from the perspective of gas emissions. The baseline emission model provided by the International Civil Aviation Organization (ICAO) was applied to estimate the landing and take-off (LTO) emissions of civil airports in China by Xia et al. [6] and to predict the future $\mathrm{CO}_{2}$ and $\mathrm{NO}_{\mathrm{x}}$ emissions of the air transportation by Owen et al. [7]. Wei et al. [8] calculated pollutant emissions of aircraft in different cruise conditions using Boeing Performance Software (BPS). Torija and Self [9] proposed an Environmental Impact Aviation Metric (EIAM) method to calculate the LTO emissions. Recently, the 2018-2019 inventory of air pollutant emissions for the Beijing-Tianjin-Hebei Airport group in China was obtained by Han et al. [10] using operational data. These studies have calculated the aircraft gas emissions from different spatial and temporal dimensions, while little research has been further conducted in terms of the temperature change caused by them. Essentially, the 
environmental impact of gas exhausted is the greenhouse effect that contributes to global warming, and the issue of global warming has attracted public extensive attention gradually [11]. In addition, it should be noted that cruise phase should attract more attention because the time spent on it accounts for the vast majority of the total, and the greenhouse effect derived from exhaust emissions is amplified at a high altitude [12].

As is widely acknowledged, aircraft exhaust emissions contributing to greenhouse effect derive from the fuel combustion in aero-engines, meaning that the acquisition of fuel consumption value is prominent in environmental impact assessment. Numerous researches on fuel consumption modeling have been carried out for its largest portion of operational costs or significant detrimental impact to environment. The methods used are mainly classified into two categories consisting of mathematical programming and machine learning. For mathematical programming methods, user manual for the Base of Aircraft Data (BADA) [13] of Eurocontrol presents a method that calculates the nominal FFR using the thrust and thrust-specific fuel consumption, which functioned by the true airspeed. And it was utilized to model the cruise fuel consumption and improved through determining new empirical constants with regard to the Mach number by Bartel and Young [14]. In the research of Senzig et al. [15], a model of fuel consumption in terminal area was proposed and verified with the consideration of temperature ratio, Mach number, and net corrected thrust. Turgut and Rosen [16] investigated an exponential relationship between fuel flow rate (FFR) and altitude for the descent phase. And there were also a series of regression models for fuel consumption estimation based on the Gaussian and $\mathrm{K}$-nearest neighbour (KNN) methods as presented by Lawrance [17]. From the studies above, the mathematical programming models are mostly based on the engine thrust and rely on a large database of aircraft performance and flight parameters with significant complexity in calculating and limited accuracy of results.

In recent decades, another data-driven method of machine learning also shows efficiency in FFR modeling. Backpropagation (BP) neural networks based on flight parameters for FFR prediction in different flight phases were presented by Liu [18]. Although the application of neural network significantly simplified the estimation process compared with mathematical programming, there were a series of defects such as its slow convergence speed and easiness to fall into local optimum. Later, Zhang and $\mathrm{Xu}$ [19] and Baklacioglu et al. [20] optimized the classical BP neural networks using particle swarm and genetic algorithm, respectively, allowing higher accuracy available, but the requirement for detailed data about aircraft operation and associated state greatly limited the practicality of the model. For example, the data of aircraft pitch angle, roll angle, and true airspeed set as input parameters in their models are always difficult to obtain in application. In this regard, trajectories parameters were employed to construct a BP neural network for FFR by Wei and Zhang [21], through the network the aircraft FFR was output given its ADS-B tracking information which was much more accessible.
Zhou [22] explored the relationship between flight altitude, ground speed, and true airspeed and developed a true airspeed estimation model, based on which a FFR model was further constructed using the deep belief network (DBN). It should be noted that the meteorological factors playing important parts in fuel consumption were excluded in their models.

In summary, there are still deficiencies in the metrics and accuracy of aviation environmental impact assessment. In order to achieve the concept of "green flight", it is practical and far-reaching to develop an effective methodology of greenhouse effect calculation for aircraft cruise, for which a calculation method based on genetic algorithm-optimized wavelet neural network (GA-WNN) is proposed in this paper. This paper contributes to aviation environmental impact evaluation from three aspects. Firstly, a supervised learning method based model considering the meteorological factors is constructed for aircraft FFR prediction, contributing to reduced dependency on detailed operation data and more conformance with the reality. Secondly, the genetic algorithm is initiatively used to optimize the architecture of wavelet neural network applied to FFR modeling, which leads to a further improvement for network performance. On top of the above improvements, a feasible and reliable methodology focused on temperature change metric for environmental impact assessment is formed, providing supports for developing sustainable civil aviation.

The reminder of this paper is organized as follows. Section 2 formulates the calculation of the greenhouse effect caused by aircraft cruise to a linear function. In Section 3, a genetic algorithm-optimized wavelet neural network model is proposed, and the developing procedure for it using real raw flight records data of the aircraft type, and Boeing $737-800$ is presented in detail. In Section 4, applications of the developed model to 9 flights are represented as numerical examples, followed by the last section, which summarizes the research with concluding remarks and gives an outlook to the future work.

\section{Problem Formulation}

For the environmental impact quantification, $\mathrm{CO}_{2}$ and $\mathrm{NO}_{\mathrm{x}}$ are considered in this paper because a vast majority of the greenhouse effect that aircraft causes when cruising comes from them. Based on their emissions and using the global absolute temperature change potential (AGTP) as a parameter, a linear function formulating the caused greenhouse effect is developed in this section.

\subsection{Greenhouse Gas Emission}

2.1.1. $\mathrm{CO}_{2}$ Emissions. According to the Emissions and Dispersion Modeling System (EDMS) published by Federal Aviation Administration (FAA), the $\mathrm{CO}_{2}$ emissions can be functioned by its emission index and FFR as follows:

$$
E_{\mathrm{CO}_{2}}=\mathrm{EI}_{\mathrm{CO}_{2}} \int_{0}^{T_{c}} \mathrm{FFR}(t) \mathrm{d} t
$$


where $E_{\mathrm{CO}_{2}}$ is the value of $\mathrm{CO}_{2}$ emissions, $\mathrm{EI}_{\mathrm{CO}_{2}}$ is the emission index of $\mathrm{CO}_{2}$ and is only relevant to the engine type, $T_{c}$ is the total time (h) spent in cruise phase, and $\operatorname{FFR}(t)(\mathrm{kg} / \mathrm{h})$ is the value of FFR at the moment of $t$.

2.1.2. $N O_{x}$ Emissions. The $\mathrm{NO}_{\mathrm{x}}$ emissions during cruise phase is also closely related to FFR and $\mathrm{NO}_{\mathrm{x}}$ emission index $\mathrm{EI}_{\mathrm{NO}_{x}}$, but the difference from $\mathrm{CO}_{2}$ emissions is that the value of $\mathrm{EI}_{\mathrm{NO}_{x}}$ depends additionally on the atmospheric conditions and FFR besides the engine type. Therefore, $\mathrm{EI}_{\mathrm{NO}_{x}}$ changes with the moment $t$ during the cruise phase.

In order to obtain the real-time $\mathrm{EI}_{\mathrm{NO}_{x}}$, a method combining interpolation using the specific FFR with modification according to atmospheric conditions is proposed in this paper, and that includes the following three steps.

Step 1: convert the FFR value in actual operational conditions to that in standard conditions (ISA, 0m) according to

$$
\mathrm{FFR}^{\prime}=\frac{\mathrm{FFR}}{\delta} \theta^{3.8} e^{0.2} M^{2}
$$

where $\mathrm{FFR}^{\prime}$ is the converted value of FFR, $\delta$ is the ratio of local atmospheric pressure to that on the standard sea level $(1013.2 \mathrm{hPa}), \theta$ is the ratio of local atmospheric temperature to that on the standard sea level (288K), $e$ is the natural constant valuing about 2.72 , and $M$ is the Mach number [8].

Step 2: get a fitting relationship between FFR and the $\mathrm{NO}_{\mathrm{x}}$ emission index in standard conditions with the use of basic emission data of engine so that the baseline $\mathrm{NO}_{\mathrm{x}}$ emission index $\mathrm{EI}_{\mathrm{NO}_{x}}^{\prime}$ can be valued from $\mathrm{FFR}^{\prime}$ through the relationship. For example, the fitting formula for CFM56-7B26 installed on Boeing 737-800 aircraft is as follows when FFR $^{\prime}$ values are between 300 and 5 000:

$$
\mathrm{EI}_{\mathrm{NO}_{\mathrm{x}}}^{\prime}=p_{1}+p_{2} \cdot \mathrm{FFR}^{\prime}+p_{3}\left(\mathrm{FFR}^{\prime}\right)^{3}+p_{4} \ln \left(\mathrm{FFR}^{\prime}\right)
$$

where $p_{1}, p_{2}, p_{3}$, and $p_{4}$ are fitting coefficients valuing $-0.0283,-2.1745 \times 10^{-7},-1.3976 \times 10^{-13}$, and 0.0055 , respectively. They are obtained by the universal global optimization (UGO) algorithm and contribute to a $R^{2}$ up to 0.9999 for (3).

Steps 3: modify $\mathrm{EI}_{\mathrm{NO}_{\mathrm{x}}}^{\prime}$ back to the value in actual operating conditions using the following equation:

$$
\mathrm{EI}_{\mathrm{NO}_{\mathrm{x}}}=\mathrm{EI}_{\mathrm{NO}_{x}}^{\prime} \frac{\delta^{0.51}}{\theta^{1.65}} \exp \left[19.0\left(0.0063-\frac{0.622 \varphi p_{v}}{p-\varphi p_{v}}\right)\right]
$$

where $\varphi(\%)$ is the atmospheric relative humidity, $p_{v}$ $(\mathrm{Pa})$ is the saturated vapor pressure, and it can be calculated through the atmospheric temperature $T(K)$ as follows:

$$
\lg p_{v}=\frac{10286 T-2148.4909}{T-35.85} .
$$

Once the emission indices at each moment $\mathrm{EI}_{\mathrm{NO}_{\mathrm{x}}}(t)$ are obtained, combined with the fuel flow rates $\operatorname{FFR}(t)$, the $\mathrm{NO}_{\mathrm{x}}$ emissions $E_{\mathrm{NO}_{\mathrm{x}}}$ can be derived as follows:

$$
E_{\mathrm{NO}_{\mathrm{x}}}=\int_{0}^{T_{c}} \mathrm{EI}_{\mathrm{NO}_{\mathrm{x}}}(t) \cdot \mathrm{FFR}(t) \mathrm{d} t
$$

2.2. Greenhouse Effect Characterization. There are several climate indicators to assess the impact of gas emissions. The absolute global temperature potential (AGTP), denoting the mean change of surface temperature caused by per unit greenhouse gases, is used in this paper to uniformly characterize the environmental impact of various gases including $\mathrm{CO}_{2}$ and $\mathrm{NO}_{\mathrm{x}}$, which can result in different temperature increases for their difference in radiative forcing and life cycles. The AGTP from gas $G$ for the time horizon of $H$ years is expressed as $\operatorname{AGTP}_{G}(H)$, and it can be calculated through

$$
\operatorname{AGTP}_{G(H)}=\left\{\begin{array}{l}
A_{G} \sum_{j=1}^{2}\left[a_{0} c_{j}\left(1-e^{-\left(H / d_{j}\right)}\right)+\sum_{i=1}^{3} \frac{a_{i} \alpha_{i} c_{j}}{\alpha_{i}-d_{j}}\left(e^{-(H / \alpha)}-e^{-\left(H / d_{j}\right)}\right)\right], \quad G=\mathrm{CO}_{2}, \\
A_{G} \sum_{j=1}^{2}\left[\frac{\alpha c_{j}}{\alpha-d_{j}}\left(e^{-(H / \alpha)}-e^{-\left(H / d_{j}\right)}\right)\right], \quad G=\mathrm{NO}_{\mathrm{x}},
\end{array}\right.
$$

where $G$ represents the exhausted gas, namely, $\mathrm{CO}_{2}$ or $\mathrm{NO}_{\mathrm{x}}$, $A_{G}\left(\mathrm{Wm}^{2} / \mathrm{kg}\right)$ is the radiative forcing change per unit $G$ causes, and $a_{0}, c_{j}, d_{j}, a_{i}, \alpha_{i}$, and $\alpha$ are all given parameters [23].

Then the surface temperature change $T(H)$ due to $\mathrm{CO}_{2}$ and $\mathrm{NO}_{\mathrm{x}}$ emissions during cruise phase can be formulated as a linear function:

$$
\begin{aligned}
\Delta T(H)= & \operatorname{AGTP}_{\mathrm{CO}_{2}}(H) \cdot E_{\mathrm{CO}_{2}}+\operatorname{AGTP}_{\mathrm{NO}_{\mathrm{x}}}(H) \cdot E_{\mathrm{NO}_{\mathrm{x}}} \\
= & \operatorname{AGTP}_{\mathrm{CO}_{2}}(H) \cdot \mathrm{EI}_{\mathrm{CO}_{2}} \int_{0}^{T_{\mathrm{C}}} \operatorname{FFR}(t) \mathrm{d} t \\
& +\operatorname{AGTP}_{\mathrm{NO}_{\mathrm{x}}}(H) \int_{0}^{T_{C}} \mathrm{EI}_{\mathrm{NO}_{\mathrm{x}}}(t) \cdot \operatorname{FFR}(t) \mathrm{d} t .
\end{aligned}
$$




\section{GA-WNN Model for Fuel Flow-Rate}

As the linear function developed previously, the aircraft FFR at each moment during cruise phase is the most critical factor for the greenhouse effect calculation. In this section, a GA-WNN model for FFR based on the wavelet neural network (WNN) technology is proposed, followed by a detailed developing procedure using the real flight records data of Boeing 737-800 aircraft.

3.1. Wavelet Neural Network. As discussed by Lai et al. [24], the wavelet neural networks (WNNs) were developed based on the technology of BP neural network and they were similar in topology. However, the original sigmoid function as the transfer function for hidden layer nodes was substituted by the wavelet basis function in $\mathrm{WNN}$, and the shift and scaling factors were introduced to replace the corresponding weights and thresholds respectively. Combined with the time-frequency locality of the wavelet analysis, the WNN has improved capability in self-learning, robustness, and adaptability, resulting in a better prediction performance.

In order to overcome the shortcomings of the previous methods that depend too much on those less publicly available data involved in airline operation details and to further improve the performance of the supervised learning based model, a three-layer WNN was constructed to model FFR of the transport aircraft during cruise phase in this paper. The WNN topology is shown in Figure 1.

In the input layer $X$, there are five neurons defined based on the aircraft state and meteorological wind factors affecting FFR in cruise phase, including the pressure altitude, the ground velocity, the heading of aircraft, and the speed and direction of the wind. With the normalized input data $h$, $v, d$, ws, and wd (see Figure 1), the output value of node $y_{j}$ in the hidden layer $Y, y(j)$ can be expressed as follows:

$$
y(j)=f_{j}\left(\sum_{i=1}^{5} w_{i j} x_{i}\right), \quad j=1,2, \ldots, J,
$$

where $J$ is the total nodes number in $Y, w_{i j}$ is the connection weight between node $x_{i}$ in $X$ and node $y_{j}$ in $Y$, and $f_{j}$ is the wavelet basis function, derived from the scaling and shift translation of a mother wavelet $\Psi_{j}$. Given a scaling factor $a_{j}$ and a shift factor $b_{j}$, the mathematical expression of $y(j)$ can be rewritten as

$$
y(j)=\Psi_{j}\left[\frac{\sum_{i=1}^{5} w_{i j} x_{i-} b_{j}}{a_{j}}\right], \quad j=1,2, \ldots, J .
$$

The neuron in the output layer $Z$ is the very factor that is going to be modeled, namely, the aircraft FFR, and its predicted value $z^{\text {pre }}$ output from the network is calculated by

$$
z^{\mathrm{pre}}=\sum_{j=1}^{J} w_{j k} y(j), \quad k=1,
$$

where $w_{j k}$ is the linked weight between node $y_{j}$ in $Y$ and node $z$ in $Z$ and $k$ takes the constant value of 1 for there are only one node in $Z$ as Figure 1 shows.

Similar to BP neural network, the WNN begins working with randomly initializing the connection weights between layers and the factors of wavelet basis function, following which the output value can be calculated forward layer by layer according to (10) and (11). Later the output error $e$ of a single simple can be written as follows:

$$
e=\left|z^{\mathrm{pre}}-z^{\exp }\right|
$$

where $z^{\exp }$ is the expected or actual value of FFR.

Through the backpropagation of $e$, the initial weights and factors are constantly corrected using a gradient learning method as (13)-(16) describe, until $e$ meets the training goal or the iteration number reaches the upper limit. The parameter of training goal is a predefined threshold. When the training error is less than this threshold, the iteration will stop even if the upper limit of iteration number is not reached:

$$
\begin{gathered}
w_{i j}(s+1)=-\eta_{1} \frac{\partial e}{\partial w_{i j}(s)}+w_{i j}(s), \\
w_{j k}(s+1)=-\eta_{1} \frac{\partial e}{\partial w_{i j}(s)}+w_{j k}(s), \\
a_{j}(s+1)=-\eta_{2} \frac{\partial e}{\partial a_{j}(s)}+a_{j}(s), \\
b_{j}(s+1)=-\eta_{2} \frac{\partial e}{\partial b_{j}(s)}+b_{j}(s),
\end{gathered}
$$

where $s$ is the iteration number and $\eta_{1}$ and $\eta_{2}$ are the defined learning rates for adjusting weights and factors, respectively.

3.2. Genetic Algorithm-Optimized Wavelet Neural Network. The gradient learning method presented above enables the network to gradually adjust along the direction of local improvement, which means inappropriate initial weights and factors can increase the possibility of falling into local optimum or even failing because of the complexity and nondifferentiability of the search space [25]. In this regard, the genetic algorithm (GA) with global search capability is employed to improve the model by optimizing the initial weights and factors in this paper. The specific steps to develop GA-WNN are organized as follows.

Step 1: sample data normalization: normalize the original sample data to an interval of 0-1 using (17) to ensure the quantization uniformity.

$$
x_{n}^{\prime}=\frac{x_{n}-x_{\min }}{x_{\max }-x_{\min }}
$$

where $x_{n}^{\prime}$ is the normalized value of simple $n, x_{n}$ is its original value, and $x_{\min }$ and $x_{\max }$ are the minimum and 


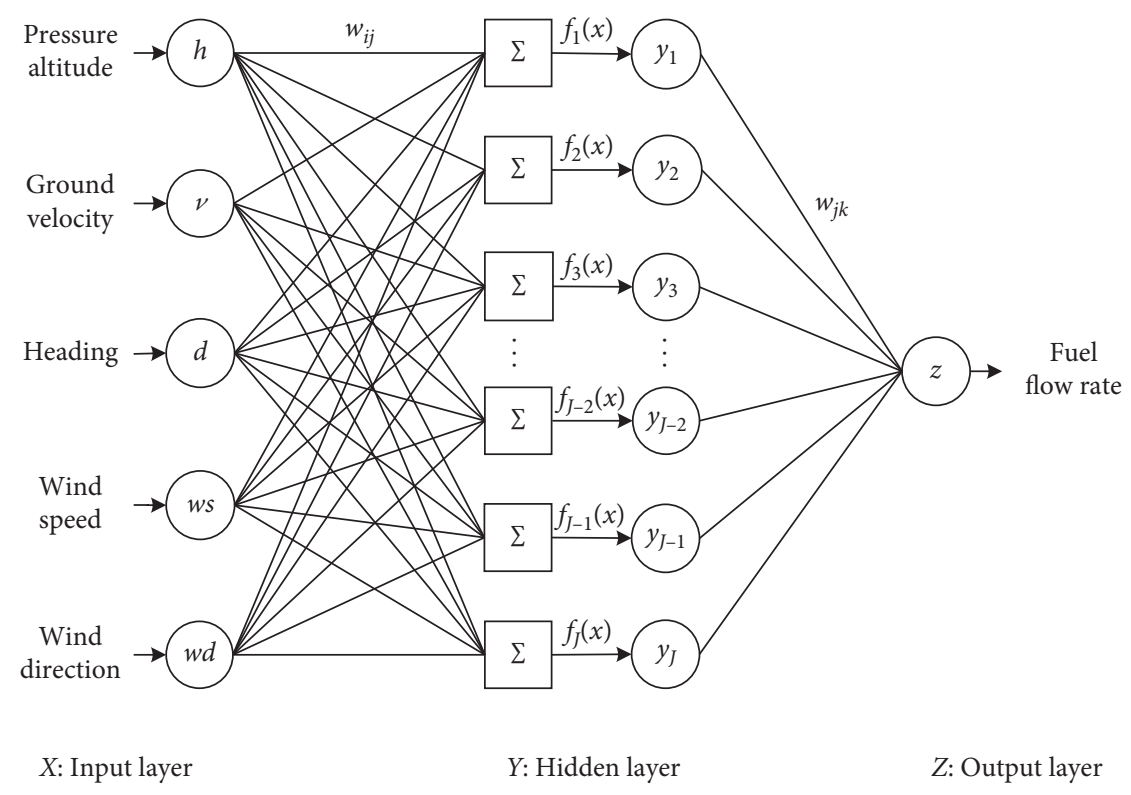

Figure 1: Topology of the WNN for fuel flow rate.

maximum value among all simples of the same category as $x_{i}$, respectively.

Step 2: specific architecture determination: use the normalized samples to further determine the architecture of the WNN, including the decision of nodes number in the hidden layer and the selection of wavelet basis functions, on which the network accuracy depends much on prediction accuracy.

Step 3: population initialization: randomly generate an initial set of solutions called population, each of which includes the connection weights for different layers and factors for wavelet basis function.

Step 4: fitness calculation: the fitness $F$ is defined as the mean squared output deviation of all samples in the testing dataset, and the function can be expressed as follows:

$$
F=\frac{1}{N} \sum_{n=1}^{N}\left(z_{n}^{\mathrm{pre}}-z_{n}^{\exp }\right)^{2}
$$

where $N$ is the sample number in the testing dataset and $z_{n}^{\text {pre }}$ and $z_{n}^{\text {exp }}$ are the output and actual FFR value of sample $n$, respectively.

Step 5: iterative evolution: update the population through evolutionary manipulations including selection, crossover, mutation, and retention, and the best individual in each generation is recorded. This step is repeated until a prespecified termination criterion.

Step 6: optimal initial weights and factors acquisition: set the optimal weights and factors according to the individual with the best fitness in Step 5 and assign them to WNN.

Step 7: prediction using GA-WNN: train the model and apply it to new data for reliable prediction results.

\subsection{Development of the GA-WNN Model}

3.3.1. Sample Processing and Division. Selected flight records data of the medium-weight transport aircraft type, Boeing $737-800$, were used in this study to develop and verify the proposed GA-WNN model. The raw data comes from several flights operating in October, 2018, and includes the information of aircraft altitude, Mach number, ground speed, and fuel flow rate of the left and right engine, which is recorded simultaneously by 4 sensors. By averaging the 4 sets, a new set was acquired and the required aircraft cruise data as samples can be extracted from it according to the operation data such as engine speeds and climb rates. Additionally, the $\operatorname{FFR}(t)$ in total was calculated by summing that of the left engine and the right one. After normalization, the samples $(2,500$ in total) were randomly split into training (60\%), testing (20\%), and validation (20\%) sets, allowing a supervised learning process to be performed.

3.3.2. Specific Architecture Determination. As is universally acknowledged, the number of neurons in the hidden layer is a significant parameter to determine the performance of neural networks, and the application of wavelet basis function distinguishes WNNs from traditional BP neural networks and also contributes to a more prospective prediction model. But it is not always better for a larger number in nodes setting and the effect of wavelets differs from each other. Therefore, how many nodes should be designed for the hidden layer and which wavelet basis function is optimal to reduce the gap between predicted outputs and expected ones are critical problems to be considered.

In order to determine the best WNN architecture, comparative tests were carried out by training the WNN for each value in the nodes number range of 6-25 in the hidden layer using different kinds of wavelet basis functions, 
respectively. The transfer functions involved in the tests include the original sigmoid function and the following 4 mother wavelets: the Morlet wavelet, the Mexican Hat wavelet (essentially the 2-order Gaussian wavelet), the 4order Gaussian wavelet, and the 6-order Gaussian wavelet, which are formulated as follows:

$$
\begin{aligned}
& \Psi(x)=\cos (1.75 x) e^{-\left(x^{2} / 2\right)}, \\
& \Psi(x)=0.8763\left(1-x^{2}\right) e^{-\left(x^{2} / 2\right)}, \\
& \Psi(x)=1.5\left(3-6 x^{2}+x^{4}\right) e^{-\left(x^{2} / 2\right),} \\
& \Psi(x)=\frac{-1}{\sqrt{2 \pi}}\left(15-45 x^{2}+15 x^{4}-x^{6}\right) e^{-\left(x^{2} / 2\right)} .
\end{aligned}
$$

With the training goal of 0.001 , each permutation was executed for 50 runs of 500 epochs with the learning rate of 0.01 for linked weights $\left(w_{i j}\right.$ and $\left.w_{j k}\right)$ and 0.001 for wavelet basis function factors $\left(a_{j}\right.$ and $\left.b_{j}\right)$. Figure 2 shows the average results of the trails.

As is shown in Figure 2, the constructed WNN performs best when 22 neurons are designed in the hidden layer using the wavelet basis function derived from Morlet wavelet, and a minimum MSE of about 0.0071 for the testing output can be obtained.

3.3.3. Optimal Initial Weights and Factors. Based on the determined specific architecture of the WNN, the mechanisms of natural selection and genetics were utilized following the steps shown in Section 3.2. For the GA implementation in this paper, the parameters and values were chosen based on the computational results in terms of accuracy during plenty of experiments. Using binary encoding, a population size of 100 was defined and a maximum number of 100 was limited for generation. The evolution manipulations were carried out with the strategies of Roulette-wheel selection, two-point crossover, discrete mutation, and elitism, and the rates for crossover, mutation, and retention were set to be $0.85,0.01$, and 0.85 , respectively. The output fitness curve of GA is shown in Figure 3. When the generation number is about 35 , there comes a converged solution and a minimum MSE approaching 0.0027 , at which time the optimal initial weights and factors can be obtained. And compared with the original minimum MSE of 0.0071 (see Figure 2), a significant improvement of optimization is well-founded.

\subsubsection{Performance Measures and Comparation Analysis.} Figure 4(a) shows the predicted and actual FFR values for 100 sample exemplars in testing dataset (500 data points in total), it is obvious that there is a good agreement between them for each sample. The output absolute percentage error (APE) of all data points in the testing dataset is counted and illustrated in Figure 4(b); it can be seen that the proportion of output with error lower than $10 \%$ is about $70 \%$, and that figure rise to $90 \%$ when the error threshold is set to be $20 \%$, preliminarily indicating an acceptable accuracy of the proposed GA-WNN model.

To further verify the reliability of the trained GA-WNN model, the validation dataset was randomly divided into 5 groups (each contains 100 data points) and they were named as G1, G2, G3, G4, and G5, respectively. As can be seen in Figure 5, the predicted FFR values closely match the actual ones in each group, demonstrating a prospective generalizability of the model.

As mentioned previously, traditional BP neural network usually uses the sigmoid function to get nodes output from input. And in its recent application on similar problems such as flight unpredictable fuel calculation [26] and residual fuel prediction [27], a linear function was also employed for this purpose. To assess the advantage of the proposed GA-WNN model for FFR prediction, its performance results were compared with those of the previous models, including BP neural networks using sigmoid (sig) or linear (lin) function to transfer between different layers. Additionally, GA-optimized BP neural networks working with different transfer functions were also compared with using the 5 groups of validation data. Note that all tests were conducted with the gradient method to learn from error so as to correct parameters during iteration. In terms of performance indicators, the mean square error (MSE), mean absolute error (MAE), mean absolute percentage error (MAPE), maximum absolute percentage error (Max-APE), and $R^{2}$ were counted and compared. Figure 6 illustrates the detailed performance results using different marked models for each data group. For example, GA-BP (sig-lin) represents a GAoptimized BP model with a sigmoid transfer function between the input and hidden layer and a linear transfer function between the hidden and output layer.

From Figure 6, it can be concluded that GA-WNN shows great advantages in any indicator for each group. Generally, the traditional BP neural networks using different transfer functions are ineffective and still questionable in reliability even after being optimized by GA for their $R^{2}$ are all less than 0.73 . The WNN performs better than them in all kinds of errors and gets a significant improvement for $R^{2}$ valuing about 0.90 in average. Furthermore, compared with the $\mathrm{WNN}$, the GA-WNN model proposed in this paper reduces various errors by more than a half, and $R^{2}$ of it reaches a reliable level up to 0.97 , which reflects its commendable performance in both accuracy and stability.

\section{Numerical Examples}

This section employs a set of numerical examples applying the GA-WNN model developed in Section 3.3 to verify the feasibility, reliability, and superiority of the proposed methodology.

4.1. Data Preparation. According to the statistical data of Civil Aviation Administration of China (CAAC), valuing about 30,452 , the number of flights operating on the route from Beijing Capital International Airport (ICAO code: ZBAA) to Shanghai Hongqiao International Airport (ICAO 


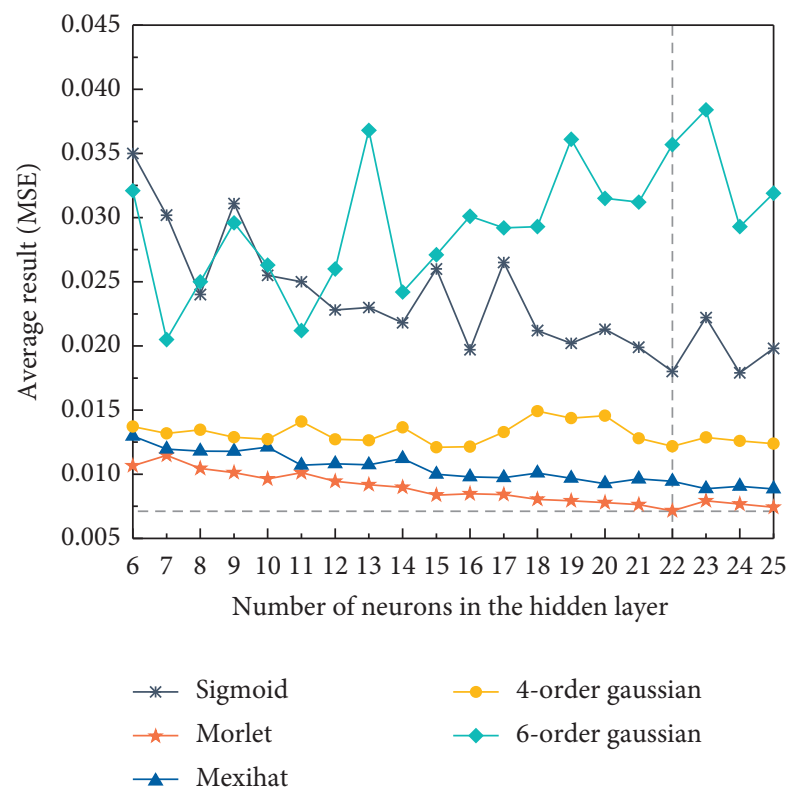

FIGURE 2: Average MSE of 50 runs using WNN with different architectures.

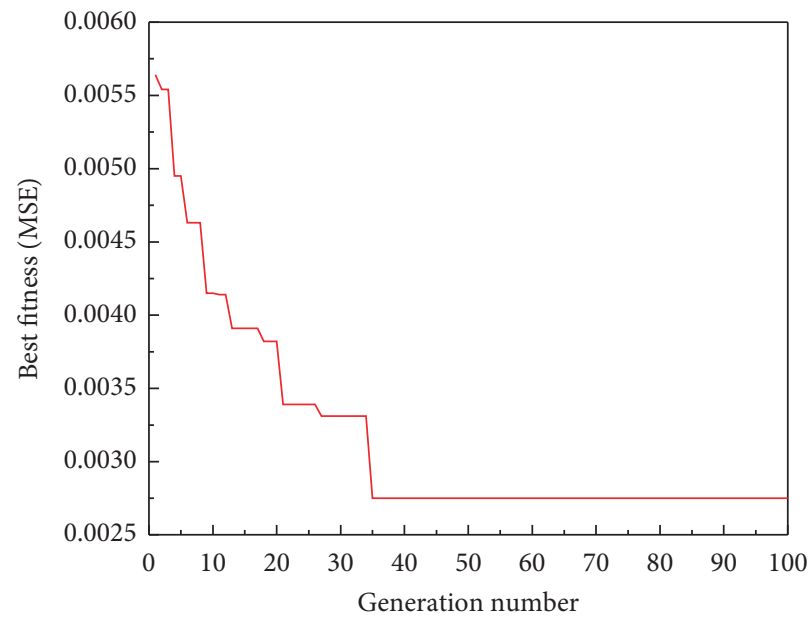

Figure 3: Testing results of GA optimization in terms of best fitness (MSE).

code: ZSSS) during the air season from March 25, 2018, to March 30, 2019, ranks the first among all domestic routes. That means ZBAA-ZSSS is the busiest flight route in China, resulting in the greatest urgency to be evaluated and controlled in regard of aviation environmental impact. Therefore, 9 flights on this route operated by Boeing 737-800 aircraft on October 2, 2019, were taken as examples.

Through the ADS-B tracking data from FlightAware on https://zh.flightaware.com/, the historical all-stage records information (including flight number, time, heading, air pressure altitude, ground speed, longitude, and latitude) of the 9 example flights were collected. Described in terms of Beijing time (UTC +8$)$, their operation durations and profile trajectories are presented in Figure 7, from which the relevant data during cruise phase can be further extracted.
According to the location information of key points on ZBAA-ZSSS shown in Table 1, the meteorological data (including atmospheric pressure, temperature, relative humidity, wind speed, wind direction, etc.) of the sounding spots around the route was gathered from University of Wyoming on http://weather.uwyo.edu/upperair/. As Table 1 shows, there are 10 segments in the route ZBAA-ZSSS in total. However, the first one from ZBAA to ELKUR and the last one from SANSA to ZSSS are for aircraft climbing and descending, respectively; only the middle 8 are for cruise phase and are considered in this paper. Using the spatial interpolation method of inverse distance weighted (IDW), the values of each meteorological parameter at different flight levels corresponding to points on the route were obtained through (23) when the three nearest sounding sites 


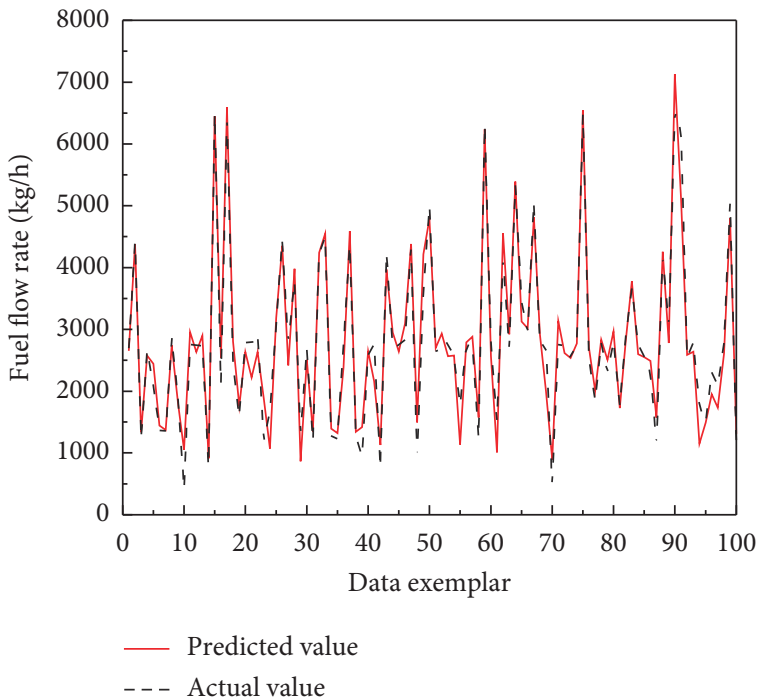

(a)

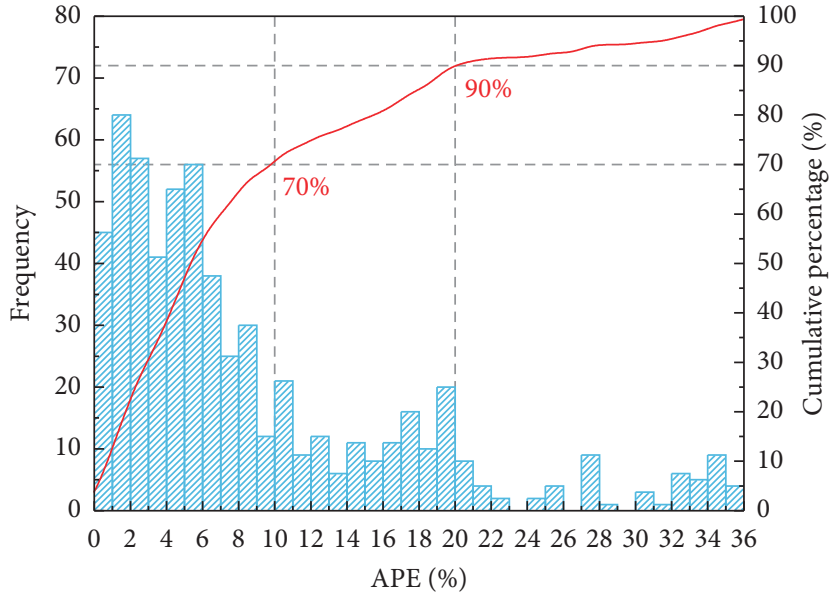

(b)

FIGURE 4: Testing results of GA-WNN model by (a) the comparison of the predicted and actual FFR values for 100 exemplars; (b) the cumulative distribution of absolute percentage error (APE) for 500 data points.

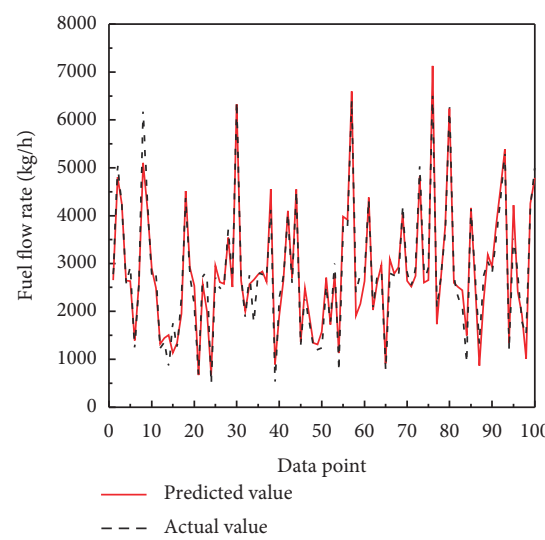

(a)

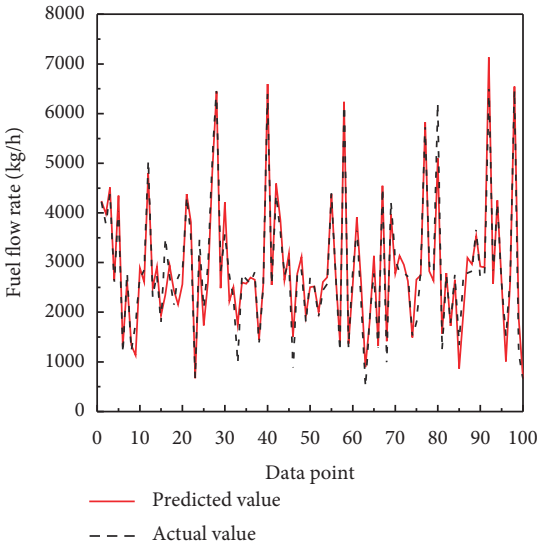

(d)

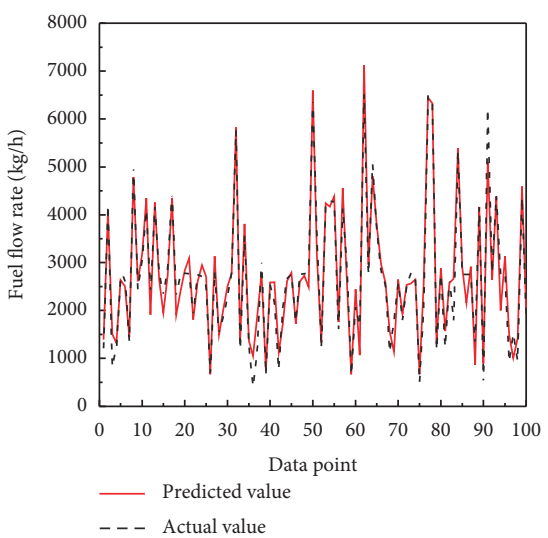

(b)

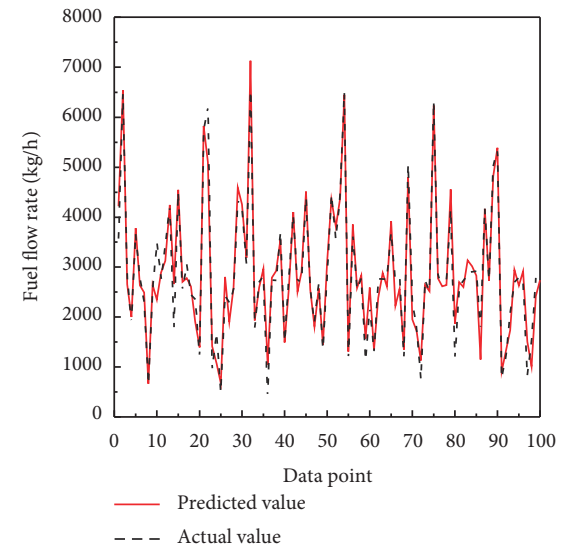

(e)

FIGURE 5: Comparison of the predicted and actual FFR values for the validation dataset of 5 groups including (a) G1, (b) G2, (c) G3, (d) G4, and (e) G5. 


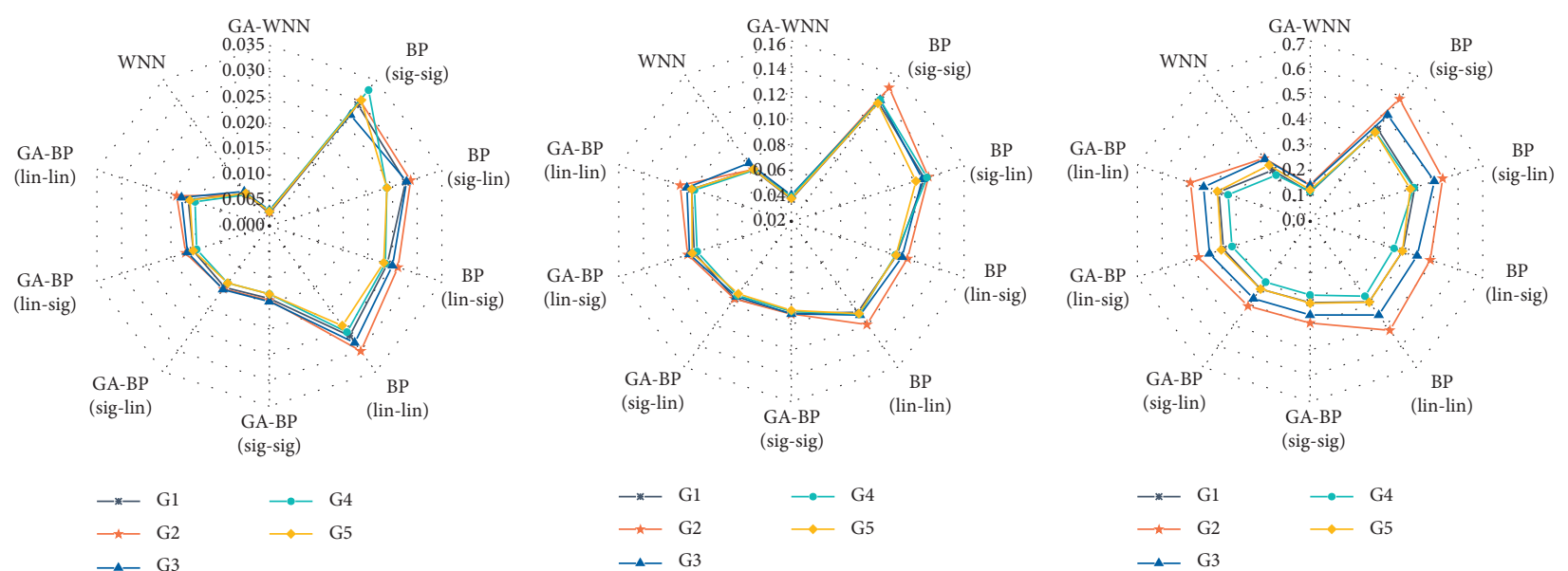

(a)

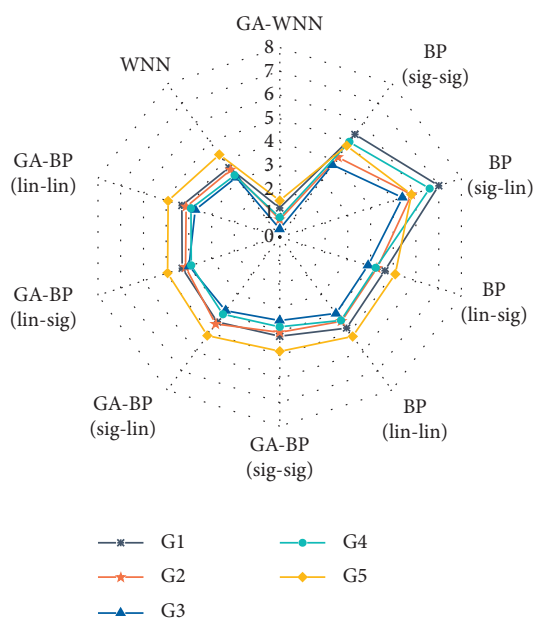

(d) (b)

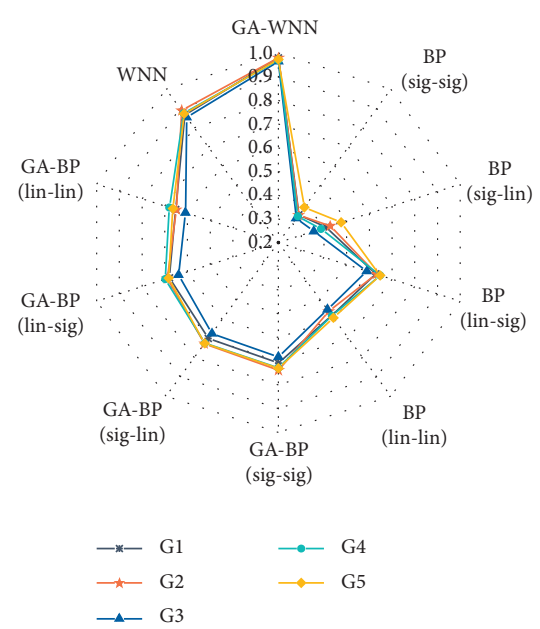

(e)

FIGURE 6: Different models performance using 5 groups of validation data in terms of (a) MSE, (b) MAE, (c) MAPE, (d) Max-APE, and (e) R2.

around were selected as samples and the power exponent was set to be 2 :

$$
R=\frac{\left(\sum_{m=1}^{3}\left(R_{m} / l_{m}^{2}\right)\right)}{\left(\sum_{m=1}^{3}\left(1 / l_{m}^{2}\right)\right)},
$$

where $R$ is the needed parameter value on the point to be interpolated; $R_{m}$ and $l_{m}$ are the collected value at the sample site $m$ and its distance away from the point to be interpolated, respectively.

After matching the trajectories information with meteorological data by latitude and longitude as well as normalizing through (17), the vector $v_{f}(t)$ for each flight $f$ at the recorded time $t$ written as (24) can be obtained and thus the input data is ready for the proposed model:

$$
v_{f}(t)=\left\{h_{f}(t), v_{f}(t), d_{f}(t), w s_{f}(t), w d_{f}(t)\right\}^{T} .
$$

4.2. Numerical Results. Applying the GA-WNN model, the FFR corresponding to each time point recorded by radar can be predicted and then the fuel consumption can be obtained through integration. Figure 8 shows the fuel consumption of different flights in each of the 8 cruising segments.

Through the total fuel consumption, the $\mathrm{CO}_{2}$ and $\mathrm{NO}_{\mathrm{x}}$ emissions during cruise phase and the resulted surface temperature change for different time horizon of 20,50 , and 100 years can be quantitatively calculated using the functions formulated in Section 2. Table 2 presents the studied flights' results.

4.3. Experimental Analysis. Using the developed methodology in this paper, the detailed results of each flight on all cruise segments can be clearly given once their trajectory data tracked by ADS-B is acquired, while, with fewer need of large amount of operation and state data, the results include the exact temperature change for different time horizons in particular. In terms of evaluation metrics and models, the proposed methodology in this paper is better in investigation depth, result accuracy, and application feasibility compared with previous ones. 


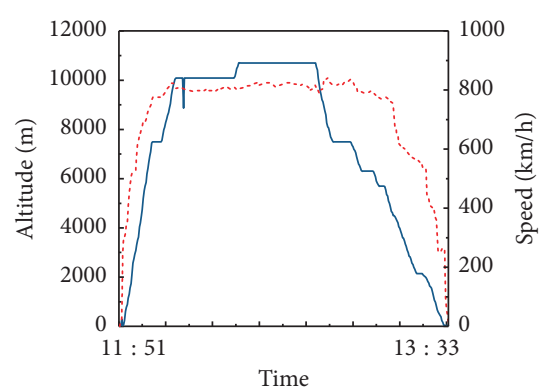

(a)

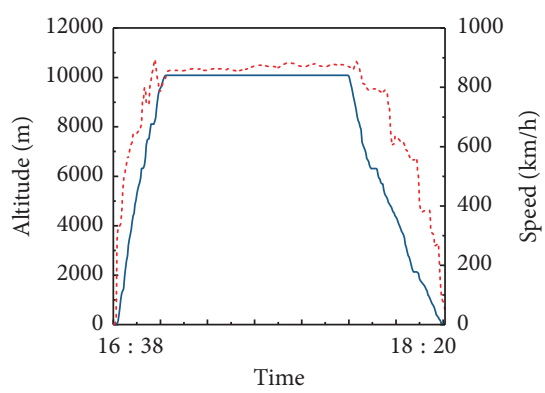

(d)

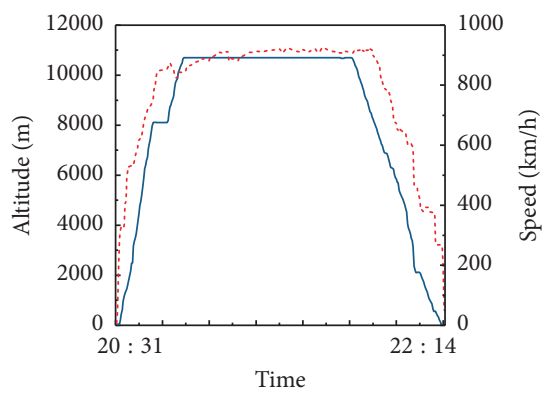

(g)

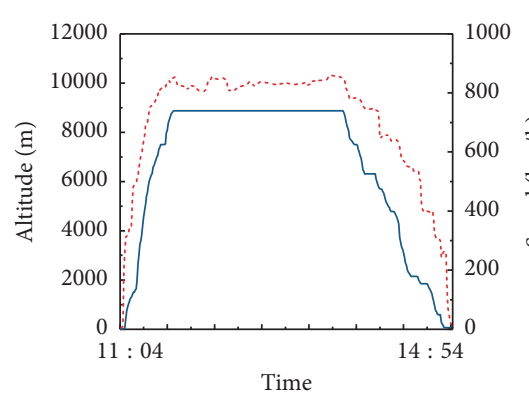

(b)

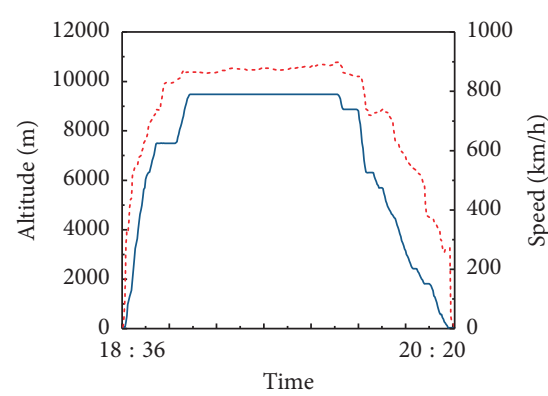

(e)

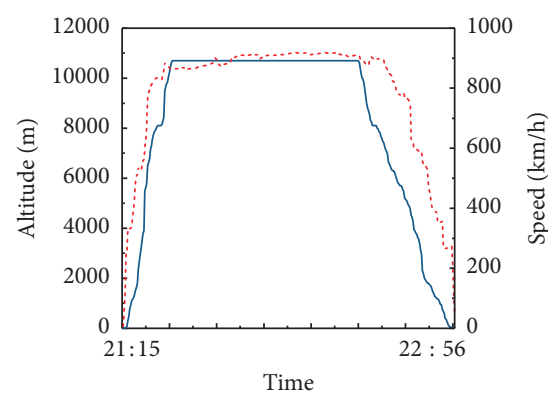

(h)

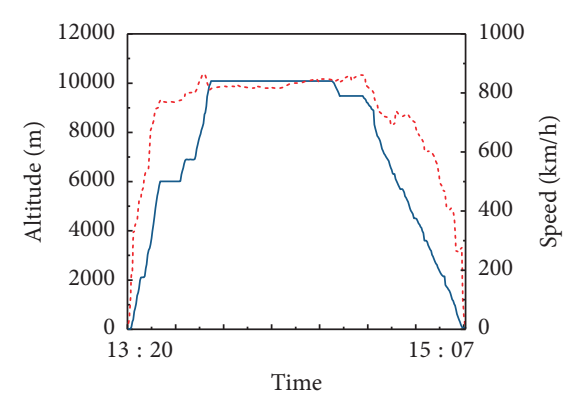

(c)

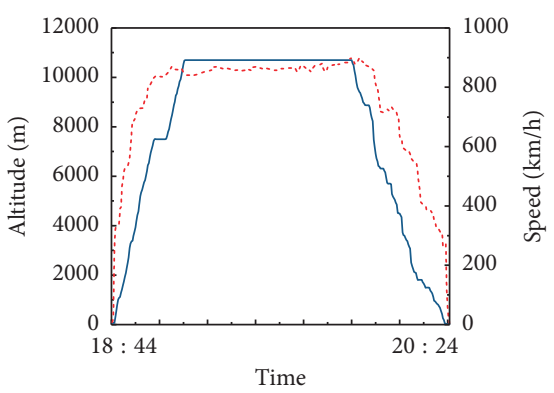

(f)

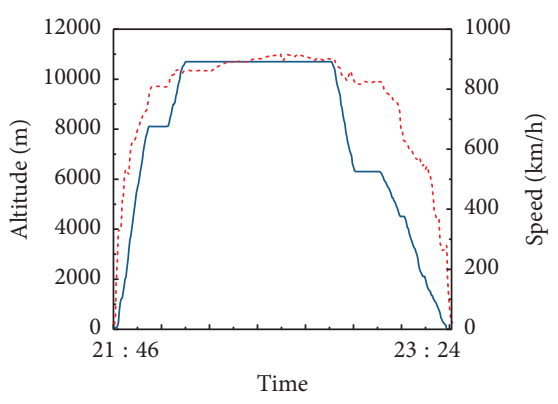

(i)

FIgURE 7: Operation durations and profile trajectories of the studied flights including (a) CCA1557, (b) CES515 R, (c) CXA8178, (d) CCA1549, (e) CSF9108, (f) CCA1885, (g) CSF9102, (h) CHH7603, and (i) CES5158.

TABLE 1: Location information of key points on the route of ZBAA-ZSSS.

\begin{tabular}{lcccc}
\hline From & To & End latitude $\left({ }^{\circ} \mathrm{N}\right)$ & End longitude $\left({ }^{\circ} \mathrm{E}\right)$ & Segment length $(\mathrm{km})$ \\
\hline ZBAA & ZBAA & 40.07 & 116.60 & 0.00 \\
ELKUR & ELKUR & 38.64 & 116.67 & 159.69 \\
OVNUG & OVNUG & 38.11 & 116.70 & 59.93 \\
GUSIR & GUSIR & 37.21 & 117.04 & 105.22 \\
ABTUB & ABTUB & 36.00 & 117.37 & 139.06 \\
UDINO & UDINO & 34.82 & 117.80 & 136.96 \\
OMUDI & OMUDI & 33.97 & 118.27 & 105.41 \\
SUBKU & SUBKU & 33.20 & 119.58 & 106.36 \\
XUTGU & XUTGU & 32.46 & 120.32 & 100.97 \\
SASAN & SASAN & 31.59 & 121.34 & 120.02 \\
\hline
\end{tabular}

From Figure 8, it can be seen that cruise flights operating on the same route with various strategies resulted in a gap about $494.69 \mathrm{~kg}$ between the minimum, $2706.94 \mathrm{~kg}$, and the maximum, $3201.63 \mathrm{~kg}$, in fuel consumption, leading to different economic benefits and environmental impact. Even for the two flights cruising on similar profile trajectories with analogous speeds and flight levels, such as CSF9102 (see Figure $7(\mathrm{~g})$ ) and $\mathrm{CHH7603} \mathrm{(see} \mathrm{Figure} 7(\mathrm{~h})$ ), there is also a significant difference in results. Apart from their discrepancy in payload, the atmospheric conditions differing with operating durations contribute a lot to the difference. This effect can be also observed from the different results of the 


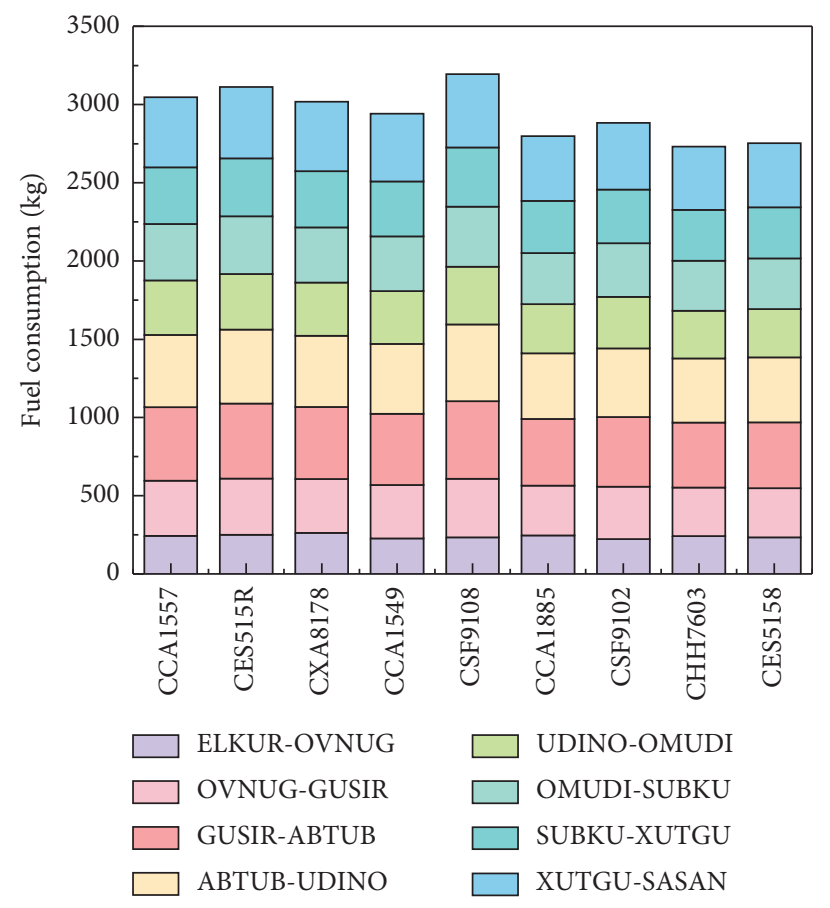

FIGURE 8: The fuel consumption of studied flights in each segment.

TABle 2: Numerical results for studied flights in terms of fuel consumption, gas emissions, and generated greenhouse effect.

\begin{tabular}{|c|c|c|c|c|c|c|}
\hline \multirow{2}{*}{ Flight number } & \multirow{2}{*}{ Fuel consumption $(\mathrm{kg})$} & \multicolumn{2}{|c|}{ Gas emissions (kg) } & \multicolumn{3}{|c|}{ Temperature change $\left[10^{-11} \mathrm{~K}\right]$} \\
\hline & & $E_{\mathrm{CO}_{2}}$ & $E_{\mathrm{NO}_{\mathrm{x}}}$ & $\Delta T(20)$ & $\Delta T(50)$ & $\Delta T(100)$ \\
\hline (a) CCA1557 & 3047.40 & 9614.55 & 43.56 & 40.88 & 7.67 & 2.49 \\
\hline (b) CES515R & 3123.07 & 9853.29 & 43.80 & 41.12 & 7.72 & 2.51 \\
\hline (c) CXA8178 & 3038.95 & 9587.89 & 42.40 & 39.81 & 7.48 & 2.43 \\
\hline (d) CCA1549 & 2942.22 & 9282.70 & 40.44 & 37.98 & 7.14 & 2.33 \\
\hline (e) CSF9108 & 3201.63 & 10101.14 & 43.50 & 40.86 & 7.69 & 2.51 \\
\hline (f) CCA1885 & 2798.59 & 8829.55 & 37.29 & 35.04 & 6.60 & 2.16 \\
\hline (g) CSF9102 & 2873.14 & 9064.76 & 37.00 & 34.78 & 6.57 & 2.16 \\
\hline (h) $\mathrm{CHH} 7603$ & 2706.94 & 8540.40 & 34.89 & 32.80 & 6.19 & 2.03 \\
\hline (i) CES5158 & 2752.32 & 8683.57 & 36.32 & 34.13 & 6.43 & 2.11 \\
\hline
\end{tabular}

segments that are almost equal in distance and operated by the same flight.

In terms of the generated greenhouse effect shown in Table 2, the global surface temperature change derives from fuel consumption but not always presents a positive correlation with it, indicating a trade-off between economic benefits and environmental contributions must be carefully considered in cruise strategies decisions.

\section{Conclusions}

In order to analyze the environmental impact and sustainability of modern air transport, a systematic methodology for calculating the greenhouse effect of aircraft cruise is presented and performed in this paper. The main research conclusions are as follows.

(1) The fuel flow rate is modeled with respect to both cruise strategies and wind factors simultaneously and developed using real flight records data based on a supervised learning method, allowing the predicted results more in line with actual operation.

(2) This paper constitutes the first attempt to optimize the initial parameters of WNN by using genetic algorithm in fuel flow-rate modeling. A Morlet wavelet and the number of 22 for hidden layer neurons are proved to be preferred and the developed GA-WNN model shows a good agreement between the predicted values and actual ones.

(3) Comparative tests with previous models conducted in this study validate that the developed model is outstanding in both accuracy and stability, with significantly reduced errors for prediction and an acceptable $R^{2}$ up to 0.97 in average.

(4) This paper employs 9 flights from ZBAA to ZSSS as numerical examples to implement the proposed methodology for greenhouse effect calculation. 
Results of each flight on all cruise segments can be effectively given and clearly compared, from which the factors affecting greenhouse effect generation and how they function can be further figured out.

With less dependency on detailed operation data, more improvements in the model accuracy, and numerical results about the exact temperature change for different time horizons, the proposed methodology in this paper performs better in investigation depth, result accuracy, and application feasibility compared with previous ones. It also supports a feasible, reliable, and superior assessment method for the performance of practical applications, such as aircraft trajectories planning, air traffic management, and operational performance assessment involved with environmental consideration. Future work can be carried out by taking contrails into account as well as exploring an optimization scheme for cruise strategies based on both economic and environmental consideration.

\section{Data Availability}

The data used to support the findings of this study are available from the first author upon request.

\section{Conflicts of Interest}

The authors declare that they have no conflicts of interest regarding the publication of this paper.

\section{Acknowledgments}

The assistance of Chen $\mathrm{Wu}$ and Jingfei Liu in data collection is gratefully acknowledged. This work was supported by the National Nature Science Foundation of China (Grant no. 61671237), and the Graduate Open Foundation of Nanjing University of Aeronautics and Astronautics (Grant no. kfjj20200735).

\section{References}

[1] Y. Tian, X. He, Y. Xu, L. Wan, and B. Ye, "4D Trajectory optimization of commercial flight for green civil aviation," IEEE Access, vol. 8, pp. 62815-62829, 2020.

[2] G. P. Brasseur and M. Gupta, "Impact of aviation on climate," Bulletin of the American Meteorological Society, vol. 91, no. 4, pp. 461-464, 2010.

[3] B. Sridhar, N. Y. Chen, and H. K. Ng, "Aircraft trajectory design based on reducing the combined effects of carbondioxide, oxides of nitrogen and contrails," in Proceedings of the AIAA Modelling and Simulation Technologies Conference, pp. 807-817, Atlanta, GA, USA, August 2006.

[4] Y. Tian, L. L. Wan, B. J. Ye et al., "Cruise flight performance optimization for minimizing green direct operating cost," Sustainability, vol. 11, no. 14, Article ID 3899, 2019.

[5] B. Sridhar, N. Y. Chen, and H. K. Ng, "Energy efficient contrail mitigation strategies for reducing the environmental impact of aviation," in Proceedings of the 10th USA/Europe Air Traffic Management Research and Development Seminar, Chicago, IL, USA, June 2013.

[6] Q. Xia, H. F. Zuo, and J. L. Yang, "Evaluation of LTO cycle emissions from aircraft in China's civil aviation airports,"
Acta Scientiae Circumstantiae, vol. 28, no. 7, pp. 1469-1474, 2008.

[7] B. Owen, D. S. Lee, and L. Lim, "Flying into the future: aviation emissions scenarios to 2050," Environmental Science \& Technology, vol. 44, no. 7, pp. 2255-2260, 2010.

[8] Z. Q. Wei, H. Z. Diao, and B. Han, "Research on calculating of aircraft pollution emissions in cruise phase," Science Technology and Engineering, vol. 14, no. 19, pp. 122-127, 2014.

[9] A. J. Torija and R. H. Self, "Aircraft classification for efficient modelling of environmental noise impact of aviation," Journal of Air Transport Management, vol. 67, pp. 157-168, 2018

[10] B. Han, W. K. Kong, T. W. Yao et al., "Air pollutant emission inventory from LTO cycles of aircraft in the Beijing-Tianjin-Hebei airport group, China," Environmental Science, vol. 41, no. 3, pp. 1143-1150, 2020.

[11] B. Płanda and J. Skorupski, "Methods of air traffic management in the airport area including the environmental factor," International Journal of Sustainable Transportation, vol. 11, no. 4, pp. 295-307, 2017.

[12] Z. F. Y. Wang, Y. Tian, L. L. Wan et al., "Progress in the study of environmental impacts of high altitude flight," Environmental Protection Science, vol. 53, no. 3, pp. 100-105, 2017.

[13] Eurocontrol, "User manual for the base of aircraft data (BADA)," 2014, https://www.eurocontrol.int/sites/default/files/field_tabs/ content/documents/sesar/user-manual-bada-3-12.pdf.

[14] M. Bartel and T. M. Young, "Simplified thrust and fuel consumption models for modern two-shaft turbofan engines," in Proceedings of the AIAA 7th Aviation Technology, Integration and Operations Conference, pp. 1450-1456, Belfast, North Ireland, September 2007.

[15] D. A. Senzig, G. G. Fleming, and R. J. Iovinelli, "Modeling of terminal-area airplane fuel consumption," Journal of Aircraft, vol. 46, no. 4, pp. 1089-1093, 2009.

[16] E. T. Turgut and M. A. Rosen, "Relationship between fuel consumption and altitude for commercial aircraft during descent: preliminary assessment with a genetic algorithm," Aerospace Science and Technology, vol. 17, no. 1, pp. 65-73, 2012.

[17] N. R. J. Lawrance, S. Sukkarieh, and B. Masson, "Using highfrequency data for predicting fuel use of jet transport aircraft," Journal of Aircraft, vol. 54, no. 6, pp. 2115-2125, 2017.

[18] J. Liu, The Aircraft Fuel Estimation Model Based on Flight Data Analysis, Nanjing University of Aeronautics and Astronautics, Nanjing, China, 2010.

[19] Y. J. Zhang and J. X. Xu, "A novel particle swarm neural network model to optimize aircraft fuel consumption," in Proceedings of the 4th International Conference on Manufacturing Science and Engineering (ICMSE), pp. 33703374, Dalian, China, March 2013.

[20] T. Baklacioglu, "Fuel flow-rate modelling of transport aircraft for the climb flight using genetic algorithms," The Aeronautical Journal, vol. 119, no. 1212, pp. 173-183, 2015.

[21] Z. Q. Wei and W. X. Zhang, "Research on constructing of matching model between fuel consumption and flight trajectories based on BP neural network," Flight Dynamics, vol. 34, no. 6, pp. 25-29, 2016.

[22] X. R. Zhou, Fuel Flow Precision Estimation Model of Civil Aviation Airplane Based on Deep Learning, Civil Aviation University of China, Tianjin, China, 2018.

[23] J. S. Fuglestvedt, K. P. Shine, T. Cook et al., "Transport impacts on atmosphere and climate: metrics," Atmospheric Environment, vol. 44, no. 37, pp. 4648-4677, 2010.

[24] Y. Lai, S. Easa, D. Sun et al., "Bus arrival time prediction using wavelet neural network trained by improved particle swarm 
optimization," Journal of Advanced Transportation, vol. 2020, Article ID 7672847, 17 pages, 2020.

[25] A. J. Tallon-Ballesteros, "Metaheuristic algorithm to train product and sigmoid neural network classifiers," Expert Systems, vol. 36, Article ID e12383, 2019.

[26] Z. Q. Wei and Y. Hu, "Unpredictable Fuel Calculation Method Based on BP Neural Network" Flight Dynamics, vol. 37, no. 6, pp. 7-16, 2019.

[27] R. P. Gu, J. H. Lai, and Z. Q. Wei, "Prediction method of flight residual fuel based on improved BP neural network," Flight Dynamics, vol. 1-6, 2020. 